\title{
ANGLICISMOS USADOS EN NARRACIONES COSTARRICENSES EN EL VOLIBOL
}

\author{
Marjorie González Gómez
}

\begin{abstract}
RESUMEN
La investigación se basa en la estrecha relación entre cultura y lenguaje. Tiene carácter lexicográfico, exclusivamente. Recopila y describe el léxico utilizado por una muestra de narradores deportivos costarricenses. El material se obtuvo de grabaciones hechas de octubre de 1988 a abril de 1989. Incluye reseñas históricas del volibol. El estado de la cuestión comprende información sobre obras lexicográficas del anglicismo en el español costarricense.

Palabras clave: Lexicografía, anglicismos, léxico deportivo.
\end{abstract}

\begin{abstract}
The research is based on a closed relationship between culture and language. It has an exclusively lexicographic character. It compiles and describes the lexicon used by a sample of Costa Rican sports narrators. The data were compiled from records done from October 1988 to April 1989. It includes volleyball historical information. The research also contains information about lexicographic works related to Anglicism in the Costa Rican Spanish.

Key words: Lexicography, Anglicisms, sports lexicon.
\end{abstract}

Marjorie González Gómez. Magíster. Profesora adjunta de la Escuela de Lenguas Modernas, Universidad de Costa Rica. San Pedro, San José, Costa Rica.

Apdo. 6372-1000 San José, Costa Rica

Correo electrónico: mggomez@ costarricense.cr

Recepción: 19-6-06

Aceptación: 20-6-06 


\section{Introducción}

\subsection{Justificación}

Se ha decidido trabajar con los anglicismos utilizados por los narradores deportivos costarricenses en el volibol, por considerar que es de importancia para las investigaciones del español en Costa Rica; interesa cuantificar y categorizar estos vocablos. Además, esta investigación no se ha llevado a cabo antes; de ahí lo apremiante de su realización.

Nos proponemos presentar un corpus de textos narrativos sobre el deporte en mención y elaborar un inventario de los anglicismos presentes en esos textos, con su correspondiente definición lexicográfica, señalamiento de etimología y establecer la tipología de esos anglicismos con un material recogido del mes de octubre de 1988 a abril de 1989.

\section{$1.2 \quad$ Objetivos}

1. Recoger un corpus representativo de narraciones en el volibol en Costa Rica, para identificar los anglicismos presentes en ese corpus.

2. Categorizar los anglicismos de acuerdo con la tipología del préstamo lingüístico siguiendo a Helmut Lüdtke.

3. Definir lexicográficamente cada uno de los rubros identificados, según el método empleado por G. Haensch de la Universidad de Ausburgo, República Federal de Alemania.

4. Contribuir en la elaboración de un nuevo diccionario del español costarricense, mediante un aporte en el área deportiva.

\subsection{Definición del anglicismo}

La Real Academia Española define el término anglicismo de la siguiente forma: "Giro o modo de hablar propio y privativo de la lengua inglesa //2. Vocablo o giro de esta lengua empleado en otra. //3. Empleo de vocablos o giros ingleses en distinto idioma". Sin embargo, estas acepciones para la presente investigación son poco precisas y no permiten llegar al punto central de nuestro propósito. Es necesario, por lo tanto, con el fin de dar un enfoque más objetivo, enmarcar el anglicismo dentro de una categoría más amplia. Esto se llevará a cabo si se analizan los anglicismos como un fenómeno, entre los muchos existentes, de lo que se ha llamado "préstamos", en los términos según los analiza Helmut Lüdtke (1974: 21-25).

\subsection{Penetración de los anglicismos}

En la historia de la lengua castellana, en lo concerniente al siglo XX, se ha marcado una gran afluencia léxica de origen en el idioma inglés. El inglés es hoy en día la más difundida de las lenguas occidentales, de ahí la influencia que ejerce en otras lenguas naturales, 
especialmente en los ámbitos de la experiencia humana que se relacionan con la ciencia, la tecnología y los deportes, razón por la cual distintas variedades de lenguaje muestran numerosos vocablos de procedencia inglesa.

Es importante aclarar que en la presente investigación se han tomado como anglicismos todos aquellos elementos que han llegado directamente del inglés, sin tomar en cuenta su etimología primigenia.

El inglés, por su condición expansiva en nuestra época, tiene una gran fuerza de infiltración en el léxico, habiendo influido también en los otros componentes de la lengua.

En el siglo XVIII y parte del XIX, el castellano tuvo gran influencia francesa. Pero el galicismo cede y comienza el reinado del anglicismo. ¿Por qué? El galicismo contaba casi sólo con el libro como única vía de penetración; el inglés, por el contrario, tiene diversos recursos, entre los cuales los más poderosos son los medio de comunicación: las agencias noticiosas, la radio, la prensa, la televisión, la industria, la ciencia, la cinematografía, los viajes, las estrechas relaciones internacionales y sociales, las competencias atléticas a nivel internacional; todo esto, sumado a la gran preponderancia económica, científica y política económica, científica y política que tienen los países de habla inglesa en el mundo entero, han contribuido a la difusión de los anglicismos. Por lo tanto, no es de extrañar, que el inglés sea hoy la lengua más difundida y casi es obligatorio mantener una relación directa e indirecta con ella (cfr Alfaro 1950: prólogo).

En el caso particular de Costa Rica, su posición geográfica ha favorecido que gran cantidad de costarricenses se desplacen por diversas razones a los Estados Unidos de América: placer, trabajo, competencias atléticas, todo lo cual redunda en una adquisición de vocablos ingleses.

En el campo conceptual que nos interesa, el de los deportes, es importante recalcar que el volibol se originó en países de habla inglesa. El volibol, de origen estadounidense (cfr Enciclopedia Salvat de los Deportes, vol. 6 1976: 433-5), fue difundido en Costa Rica por Don Alfredo Cruz, a su regreso de los Estados Unidos, en la década de los cincuenta (cfr $V$ Copa Centroamericana Mayor de Voleibol 1989: 4-6).

El deporte ha sido, sin duda, una fuente prolífera de anglicismos. De las modernas agencias noticiosas, las más grandes pertenecen a países de habla inglesa, transmiten los acontecimientos atléticos más importantes y, en muchos casos, los mensajes son recibidos en inglés por traductores que, presionados por el tiempo y sin atender cuidadosamente los usos castellanos transmiten la información por medio de textos portadores de elementos lingüísticos de la lengua originaria. De esta manera, muchos anglicismos se difunden e incorporan en el español: léxico, campo semántico y construcciones anglicadas.

\subsection{Reseña histórica}

\subsubsection{Orígenes del volibol}

El volibol se comenzó a practicar en 1895, en la Asociación de Jóvenes de Holyoke (E.E.U.U.). Su creador, William G. Morgan, profesor de educación física del movimiento Y.M.C.A. (Asociación de Jóvenes Cristianos), había formulado el juego para aquellos muchachos para quienes el baloncesto resultaba un deporte demasiado fuerte. Lo denominó primitivamente "Mintonetti" y el nombre actual de "Volley-Ball" se debió al profesor A.T. Halsted de la Universidad de Springfield. 
Un año después de su creación, aparecieron publicadas en la revista Physical Education las primeras reglas de este juego, pero es en 1938, tras varias rectificaciones, cuando se establecen las normas del actual volibol.

A pesar de la rápida expansión que alcanzó en los Estados Unidos, su reconocimiento como deporte atlético se realizó en Europa; fue en París, con motivo de la creación de la F.I.V.B. (Federación Internacional de Voleibol) en 1947, en donde quedaron redactadas las primeras reglas internacionales. Las posteriores modificaciones y mejoras, tanto para facilitar el arbitraje como para obtener un juego más lucido, están a cargo de la Comisión de Leyes de Juego. Estas modificaciones, que se realizan con extremada prudencia, han de ser aprobadas por el congreso de la F.I.V.B., que se convoca cada cuatro años y coincide con la Olimpiadas. En esta Federación Internacional se hallan integrados 987 países, entre los que sobresalen la U.R.S.S. y China por el número de practicantes.

En el continente americano, el volibol obtiene una rápida expansión; se halla completamente integrado en todos los países y se incluye en competencias de importancia, como son los Juegos Panamericanos.

Su introducción en otros países se efectuó por dos medios: en el continente asiático, fueron los misioneros americanos los encargados de difundirlo; mientras en el europeo, lo hicieron los soldados estadounidenses participantes en la Primera Guerra Mundial. Durante muchos años se jugó, especialmente en Europa, como un juego de recreo para practicar en la playa sin reglas fijas (cfr Enciclopedia Salvat de los Deportes. Vol. 6. 1976: 433-5).

\subsubsection{Historia del volibol en Costa Rica}

Para el año 1935, donde hoy se encuentra el Colegio Napoleón Quesada, estaba el antiguo Club Deportivo Alemán. Allí se practicaba una especie de juego, en el cual se elevaba un mecate a cierta altura y mediante golpes se hacía pasar una bola de un lado a otro; esto se considera el primer movimiento de volibol en Costa Rica. Para 1938, se realizaron los Juegos Centroamericanos del Caribe y Panamá, a los cuales los costarricenses tuvieron la oportunidad de asistir con la selección de baloncesto. Estos observaron un juego oficial de volibol, que los incitó a traerlo a Costa Rica y a jugarlo en forma recreativa. Así fue la introducción del volibol de Costa Rica.

En 1942, el ejército de los Estados Unidos difundió este deporte en todos sus campos militares, dado el costo tan barato de los implementos. Don Alfredo Cruz se encontraba en los Estados Unidos y aprendió a jugarlo ahí. A su regreso a Costa Rica, fundó el Instituto de Educación Física, en donde introdujo la enseñanza del volibol y realizó un campeonato por parejas en el Liceo de Costa Rica. A partir de ese año, empezó su difusión en todo el país. En 1952, el Dr. Rodrigo Pacheco López y el profesor Alfredo Cruz Bolaños difundieron aún más este deporte, y así se ve un primer partido femenino entre el Colegio de Sión y el Colegio Lincoln, en donde sobresale la señorita Mary Jackson.

En 1964 y 1965, se funda la Asociación Nacional de Volibol, cuyo primer presidente fue don Carlos Luis Monge.

Luego, en 1970, con ocasión de los XI Juegos Centroamericanos y del Caribe en Panamá, nuestro país asiste con la selección masculina, lo que constituyó toda una experiencia para el volibol nacional. 
A partir de 1965 y hasta 1971, funciona la Asociación Nacional de Volibol; pero en este último año muere por falta de tiempo de sus organizadores. En 1972, ya no se organizó ninguna clase de campeonato ni actividades de volibol a nivel nacional, pero los equipos siguieron entrenando y ejerciendo presión sobre los directivos y organizadores para reanudar las actividades.

En 1973, regresa de Alemania el profesor William Corrales, quien, ante la presión que ejercen los diferentes equipos, decide organizar un campeonato, por invitación, en setiembre de 1973 en el Gimnasio de la Universidad de Costa Rica. El comité organizador estaba formado por William Corrales, Luis Castro, Jorge Muñoz, Orlando Abellán, Carlos Weeden, Fabio Goñi y Carlos Sánchez.

El volibol tiene día tras día mayor cantidad de practicantes. Para 1976, nuestro país organiza la II Copa Centroamericana de Volibol a nivel mayor, la cual fue todo un éxito. Posteriormente, la participación internacional se incrementa, especialmente en el nivel femenino. Es así como se participa en el Norceca Juvenil 1976, en el que se clasifica para participar en el Primer Mundial Juvenil, el cual se realizó en Brasil en 1977 y en donde se obtuvo el octavo lugar. Las mujeres luego viajaron a los Juegos Centroamericanos y del Caribe 1978, realizados en Medellín, en donde obtuvieron el sétimo lugar. Luego se asistió, en 1977, a la III Copa Centroamericana en Guatemala y a los II Juegos Centroamericanos en El Salvador. Debido a la situación política en el área centroamericana, la participación internacional decrece. A nivel nacional, las cosas tampoco marchan bien: la DEFYD (Dirección de Educación Física y Deportes) nombra una comisión reestructuradora, la cual toma las riendas del volibol nacional; y normaliza la situación.

En 1981 se reanudan los contactos internacionales: el volibol femenino es invitado a participar en el II Mundial Juvenil en el cual se obtiene el 15avo lugar. Ese mismo año se participa en la Primera Copa Juvenil en Guatemala, y en ese mismo país también se participa en la Copa de Santo Domingo, cuando por primera vez se ganó un "set" a la selección de la República China (Taiwán). Para 1982, nuestro país organizó la primera Copa Centroamericana Infantil. Después de ese año, los intercambios disminuyeron y no fue sino hasta los III Juegos Guatemala 85-86 cuando se reiniciaron regularmente las competencias en los niveles juvenil y mayor, hasta llegar a la V Copa Centroamericana 1989 que se celebró en San José (cfr V Copa Centroamericana 1989: 2-4).

\section{Estado de la cuestión}

Las investigaciones sobre el componente léxico del español en Costa Rica tienen una tradición de por lo menos un siglo, habiendo sido efectuadas, en su mayoría, con criterios preceptivos (Sánchez Corrales 1988).

En lo que concierne al anglicismo, no ha habido trabajos significativos, con excepción de la obra de Zúñiga Tristán (1976), El anglicismo en el habla costarricense, que, en opinión de Sánchez Corrales (1988),

Es un inventario amplio de anglicismos que muestra la erudición de la autora. Si bien la investigadora dice que su estudio se basa en "el lenguaje oral de nuestro pueblo" (Zúñiga 1976: 15), al no emplearse con rigurosidad criterios de frecuencia, productividad, riqueza semántica, distribución geográfica (Salas y otros, 1977), la investigación tomada en su conjunto produce la impresión de que el español en Costa Rica es anglicado por excelencia, lo cual es falso. 
Igualmente, existen unos artículos publicados por don Cristian Rodríguez en la Página Quince del periódico La Nación entre los años 70 y 82, y la tesis de la licenciada Zuray Mora Cárdenas (1985): Cristian Rodríguez, los anglicismos y el español de Costa Rica, en la cual la autora investiga el aporte crítico que don Cristian Rodríguez hizo al español de Costa Rica, con el fin de contribuir con una recopilación de todo ese valioso material publicado durante casi treinta años en varios periódicos locales, especialmente La Nación. Es importante notar que en la tesis de la licenciada Mora Cárdenas no aparece ninguno de los anglicismos encontrados en las narraciones deportivas, lo cual demuestra que la presente investigación es un aporte importante para aquellos que estudian el español, además de ofrecer una opción para continuar esta clase de estudios en Costa Rica. Por los demás, lo único que existe son artículos esporádicos en la prensa.

Como puede notarse, no hay ningún trabajo sobre el aporte léxico del inglés en el español en Costa Rica, en el campo del volibol.

\section{Marco teórico}

La elaboración del presente diccionario lexicográfico se hizo siguiendo la metodología de Günter Haensch y su equipo de léxicografos de la Universidad de Ausburgo, República de Alemania, tal como la propone para la organización del Nuevo Diccionario de Colombianismos, fase piloto del proyecto Nuevo Diccionario de Americanismos (cfr G. Haensch 1982), que se hace en esa universidad. Se sigue, además, la tipología del préstamo según la teoría de Helmut Lüdtke.

El método Günter Haensch es netamente pragmático y ha sido complementado con las ideas teóricas de Secco (1987). A continuación, se explica la terminología empleada en tal método.

Entrada: unidad léxica que es objeto de explicación. Se utiliza el rubro "palabra" en el sentido en que la gramática tradicional entiende este término (cfr. Nuevo Diccionario de Americanismos instrucciones para la Redacción, sin publicar). Las entradas pueden estar formadas por una sola palabra, o sea unidades léxicas univerbales, o por construcciones léxicas pluriverbales formadas por un conjunto de palabras, por ejemplo, set average y bola fer.

Lema: voz guía que encabeza el artículo, se ordena alfabéticamente, conforma la macroestructura del diccionario; se escribe con minúscula, a menos de que las reglas de ortografía exijan lo contrario.

Artículo: la totalidad de la información lexicográfica, contenida bajo un lema, incluido el mismo lema.

Etimología: al final de artículo se da la etimología que informa sobre el origen del lema. Si bien no se han buscado correspondencias fonológicas para identificar la etimología, el uso del vocablo castellano en la misma acepción que tiene en inglés y la semejanza fonética en la pronunciación han sido los criterios fundamentales para la identificación de la etimología.

Definición: es el corazón del artículo lexicográfico, lo que permite hablar de un diccionario per se. La definición lexicográfica se diferencia de la definición tal como se entiende en la lógica moderna, por su naturaleza de una explicación semántico pragmática. Esto es:

a) Un análisis o descripción del contenido semántico básico de cada unidad léxica tratada en el diccionario. 
b) Una aclaración sobre el uso operacional de la unidad léxica tratada, ya sea de su función sintáctica o de la forma particular en que se emplea (cfr Haensch 1982: 5).

De acuerdo con lo anterior, puede haber tres tipos generales de definiciones; nosotros definiremos únicamente las dos que se utilizan en esta investigación. El tercer tipo de definición, la descripción, no es pertinente a los objetivos del presente trabajo, ya que, además de la información semántica, especifica aspectos enciclopédicos. Nos interesan, pues, la paráfrasis y la instrucción operacional.

1. Paráfrasis: definición basada en el análisis semántico, cuyo objetivo es señalar los rasgos distintivos del significado.

2. Instrucción operacional: aclara la función sintáctica, es decir, el uso pragmático de una unidad léxica en el contenido de la comunicación.

Si la definición cumple con lo anteriormente mencionado, debe ser capaz de sustituir el término definido sin alterar su significado, y sin que interfiera el hecho de que lo definido sea un lema univerbal o un sintagma pluriverbal. Si se cumplen los requisitos de la definición, se podría establecer que esta es en realidad un sinónimo de lo definido. La sinonimia, sin embargo, no es intercambiable en todos los contextos; en algunos casos, da una equivalencia de la relación connotativa, pero no de la denotativa. Por lo tanto, la definición no puede ser absoluta; es sólo una aproximación a la igualdad.

Hecha la aclaración, podríamos decir que la sustitución es, por lo tanto, la prueba fundamental para una definición. "Si el enunciado definidor puede sustituir al término definido en un enunciado de habla sin que el sentido objetivo de éste se altere, el enunciado definidor es válido" (M. Seco 1987: 21).

Como resultado de la ley de sinonimia está la identidad categórica entre lo definido y la definición. Si lo definido es un nombre sustantivo, la definición está constituida por otro nombre o por una construcción sustantiva. Si lo definido es un adjetivo, la definición es un adjetivo léxico con especificadores o sin ellos, o una proposición adjetiva o de complemento preposicional. Cuando se trata de verbos en forma infinitiva, la definición la da otro verbo también en forma infinitiva, seguido o no de complementos.

En cuanto a la forma, la definición se inicia siempre con mayúscula, excepto cuando se inicia con una palabra que exige remisión.

\subsection{Metalenguaje de la definición}

El metalenguaje utilizado en las definiciones pertenece al español estándar; es básicamente neutro, de manera que sea comprensible para los diferentes hablantes de la lengua española. Si en el texto, al definir, se emplean préstamos del inglés para lograr una explicación semántico-pragmática más precisa, se usa la remisión.

Sinónimos: al ser la definición un análisis o descripción del contenido o del uso del una unidad léxica y no su equivalente, se excluye el uso de definiciones por medio de sinónimos para evitar caer en un círculo.

Los sinónimos se dan como una explicación adyacente a la definición dada; van encerrados entre corchetes, se escriben en negrita y se colocan al final de la definición y antes de la etimología. 


\subsection{Estructura de los artículos}

Orden de las acepciones: cuando un lema univerbal tiene más de una acepción, estas se separan entre sí por medio de una raya horizontal sencilla (/) y con un número arábigo subrayado. Si se trata de acepciones de una unidad léxica pluriverbal, se enumeran por medio de una letra del alfabeto en minúscula.

\subsection{Colocación de las unidades pluriverbales}

Las unidades pluriverbales, por ser compuestas, van siempre subordinadas al lema que les da entrada en el artículo. Se escoge entre los elementos que la componen uno que actúe como lema, al cual se le llama elemento lema, que actúa única y exclusivamente para darle entrada en el artículo.

\section{Metodología}

\subsection{Grabaciones}

Para recoger el corpus lingüístico, se procedió a grabar doce partidos de volibol, con un resultado de mil veinte minutos (1.020) de grabación. Las grabaciones se hicieron cuando se disputó el campeonato nacional.

\subsection{Procesamiento de material}

Una vez hechas las grabaciones, se procedió a su transcripción textual, incluidos comentarios y entrevistas.

En este corpus, se procedió a identificar los anglicismos utilizados por los narradores costarricenses; luego se examinó si estaban registrados en diferentes diccionarios: Real Academia Española, Diccionario de la Lengua Española, Diccionario crítico etimológico castellano e hispano, Diccionario de Autoridades y otros. Igualmente, se buscaron en algunos diccionarios bilingües que aparecen en la bibliografía, para las correspondientes aclaraciones etimológicas.

Identificados los anglicismos, se procedió a elaborar la correspondiente definición lexicográfica y la categorización tipológica.

\subsection{Marcas metalingüísticas}

Las marcas metalingüísticas constituyen el conjunto de siglas y símbolos que aclaran y definen aspectos de la unidad léxica.

\subsubsection{Marcas gramaticales}

Un diccionario es una obra de consulta que define o aclara, fundamentalmente, el significado, de manera que la categoría gramatical parece no ser indispensable, pues la lengua es independiente de la existencia del diccionario. Sin embargo, un diccionario sin marcas gramaticales es inconcebible, de manera que hay que partir del concepto de la lengua en sí con sus categorías y reglas, para entender la organización del registro léxico. 
Las siguientes son las marcas gramaticales usadas en este diccionario, las cuales se han colocado inmediatamente después del lema.

$\begin{array}{ll}\underline{\text { Adj }} & \text { adjetivo } \\ \underline{\mathrm{m}} & \text { sustantivo masculino } \\ \underline{\mathrm{f}} & \text { sustantivo femenino } \\ \underline{\mathrm{v}} & \text { verbo }\end{array}$

\subsection{Abreviaturas}

Las abreviaturas permiten el ahorro de espacio, con lo que se limita el volumen y se puede, de esta manera, incluir más información léxica o explicaciones.

A continuación se especifican las abreviaturas empleadas en este trabajo:

DRAE Registrado en el Diccionario de la Real Academia Española

vb Volibol

\subsection{Signos tipográficos}

$\quad$ Tilde. Se usa para sustituir al lema en forma exacta en las unidades pluriverbales.

* Asterisco. Introduce unidades pluriverbales subordinadas a un lema autónomo, y las separa del conjunto de acepciones que le corresponden.

$\rightarrow \quad$ Flecha. Se usa para indicar la remisión.

/ Barra oblicua. Separa las diversas acepciones de una unidad univerbal.

[ ] Corchetes. Encierran sinónimos.

: Dos puntos. Separa el elemento-lema de la unidad léxica pluriverbal a la que se le da entrada.

, Coma. Separa los sinónimos de un mismo lema.

// Barra doble. Separa las unidades pluriverbales entre sí.

\subsection{Números arábigos}

Se usan los números arábigos dentro del artículo para separar las diversas acepciones de un mismo lema.

\subsection{Letras minúsculas}

Se utilizan para identificar las acepciones correspondientes a una misma unidad pluriverbal.

\subsection{Uso de la negrita y del subrayado}

Aparecen con negrita los lemas, las unidades léxicas a las que se remite, los sinónimos y la etimología.

Se subrayan las marcas metalingüísticas, por ejemplo, adj por adjetivo; las abreviaturas, por ejemplo, v $\underline{b}$ por volibol; y los números arábigos. 


\section{El préstamo lingüístico}

Los préstamos están unidos inseparablemente por su naturaleza a la esencia y cambio posterior de toda lengua. Sus causas se pueden encontrar en la necesidad comunicativa que todo individuo posee; también como resultado de nexos históricos, socio-políticos, geográficos; sobre todo, por la necesidad de comunicación que existe entre los habitantes. Como lo indica Martínez Hernández (1974: 7) en el prólogo del libro Historia del léxico románico:

"La sucesión histórica de las distintas situaciones lingüísticas se ofrece como resultado de profundas conmociones políticas y culturales. Pensemos, por ejemplo en lo que para la renovación del léxico significa el Cristianismo, la invasión de los bárbaros o el Renacimiento. Es un mundo de continuos cambios. Los vocablos pasan de unas lenguas a otras en virtud de "préstamos". Y no solo se prestan materias, sino procedimientos formales y hasta matices de sentido. Cada palabra, además de atenerse a ciertas leyes, puede tener su historia particular. Hoy día, ¿cuántos préstamos deberemos al inglés? Y los esqueletos de las palabras, que no otra cosa son las siglas, ¿no nos cercan por todos los lados, gesticulantes? Nada inventamos: son dos características de nuestro léxico actual".

En la presente investigación se usará como fundamento el concepto de "préstamo" que describe Helmut Lüdkte, en su libro Historia del léxico románico. Según Ludtke, el préstamo lingüístico ocurre cuando: "dadas dos lenguas A y B, A influye léxicamente en B o viceversa" (1974: 22). Al hablar de préstamos, según él, se comprenden los conceptos de: préstamos de vocabulario, préstamos de formación y préstamos semánticos, conceptos que se desarrollarán en el capítulo siguiente.

\section{Tipología de los anglicismos en narraciones deportivas costarricenses}

\subsection{El préstamo de vocabulario o léxico}

En el préstamo de vocabulario se conserva tanto como sea posible la forma fónica de la palabra. Se dice tanto como sea posible, porque la reproducción exacta de una palabra extranjera es casi imposible de lograr.

\subsubsection{Préstamos de vocabulario propiamente dicho}

$\begin{array}{ll}\text { Préstamo } & \text { Étimo } \\ \text { ausay } & \text { outside } \\ \text { aut } & \text { out } \\ \text { averaje } & \text { average } \\ \text { couch } & \text { coach } \\ \text { couchada } & \text { couching } \\ \text { escor } & \text { score } \\ \text { espor } & \text { sport } \\ \text { están } & \text { stand } \\ \text { faol } & \text { foul } \\ \text { faul } & \text { fault } \\ \text { net } & \text { net } \\ \text { réferi } & \text { referee }\end{array}$




$\begin{array}{ll}\text { repris } & \text { reprise } \\ \text { riley } & \text { relay } \\ \text { set } & \text { set } \\ \text { set averaje } & \text { set average } \\ \text { suceso } & \text { success } \\ \text { volibol } & \text { volleyball }\end{array}$

\subsection{Préstamos de raíces}

Consideramos necesario distinguir entre préstamos de vocabulario propiamente dicho y préstamos de raíces, en virtud de que entre ambos casos se establece una distinción a partir de aspectos morfológicos.

Los siguientes vocablos constituyen una subcategoría de los préstamos de vocabulario léxico; una raíz, tomada del inglés, mediante los procesos de derivación castellana, adquiere la condición de palabra castellanizada.

Término

español

couchada

faulear

\section{Raíz}

coach + secuencia de sufijos $-\mathrm{a},-$ da

foul + secuencia de sufijos $-e,-a,-r$

\subsection{Préstamo de formación}

En este tipo de préstamo se agrupan todas aquellas palabras que, influenciadas por un modelo extranjero, en este caso el inglés, han sido recreadas a partir de elementos lingüísticos propios. En esta categoría hay tres tipos: calcos de esquema, calcos un poco más libres frente al modelo y el préstamo de creación (Ludtke 1971: 23). En el corpus de la presente investigación, se encontraron únicamente calcos de esquema.

\subsubsection{Calcos de esquema}

En esta categoría la traducción es exacta, se traduce palabra por palabra; el único cambio es que en algunos casos se invierten los miembros, de acuerdo con la morfología castellana.

balonvolea volleyball

\subsection{Préstamo semántico}

El tercer tipo de préstamo, según Ludtke, es el semántico: préstamos de significado. En esta categoría encontramos palabras que ya existen en castellano y, por influencia extranjera, amplían su significado con una acepción completamente diferente a la que tienen en esta lengua. Esa nueva acepción ha sido tomada de la lengua donante, el inglés en el presente caso, como por ejemplo:

$\begin{array}{ll}\text { evento } & \text { event } \\ \text { servicio } & \text { service } \\ \text { suceso } & \text { success }\end{array}$

De los tres préstamos anteriores, podemos notar que hay una semejanza de significantes, lo cual habría facilitado la ampliación semántica de los vocablos castellanos. 


\section{Diccionario}

\subsection{Aspectos generales}

El presente diccionario consta de ciento cuatro (104) entradas, cuya redacción se ha efectuado según el método lexicográfico de Haensch. Es un diccionario lingüístico, relativo a un micro campo de la experiencia humana en el volibol.

\subsection{Inventario léxico}

A

aut $\underline{\mathrm{m} 1}$ Lugar fuera de las líneas que delimitan el área de juego. / 2 Sanción que decreta el árbitro del juego cuando la bola sale de los límites de la cancha. [fuera]. De out.

averaje $\mathrm{m} 1$ Tanto por ciento que se saca en el volibol, conciente de los $\rightarrow$ sets ganados entre los perdidos. De average.

B

bloqueo $\underline{m}$ Obstáculo que ponen los jugadores al equipo contrario para evitar que hagan jugadas o que anoten puntos. Derivado de $\rightarrow$ bloquear, y este de block.

C

contusión $\underline{m}$ b Golpe que recibe un jugador durante el juego. [lesión] Derivado de contusionar y este de contusion.

contusionado adj Golpeado durante el juego. Derivado de contusionar y éste de contusion.

contusionar $\underline{\mathrm{v}}$ Causar daño físico por medio de un golpe [lesionar]. De contusion.

couch $\underline{m} \quad$ Miembro del equipo cuya misión es dirigir y aconsejar a los jugadores. De coach.

couchada $\underline{f}$ Instrucción técnica dada a los jugadores por su entrenador. De coach.

$\mathbf{E}$

escor $\underline{m} 1$ Número de puntos obtenidos por un equipo. $/ 2$ Tablero en donde se anotan los puntos obtenidos por los equipos que están jugando [marcador]. De score.

espor $\underline{m} \quad$ Palabra que se usa para denominar todo lo que conlleva ejercicio físico [atletismo, ropa deportiva]. De sports.

están $\underline{m}$ Gradería con bancos para los jugadores que no están jugando [gradería, banca]. De stand.

evento $\underline{m} \quad$ Espectáculo atlético [encuentro]. De event. 
F

faul m 1 Sanción decretada por el árbitro por faltas cometidas [falta]. / 2 Falta que comete el jugador al golpear y lanzar la pelota fuera de los límites reglamentarios del terreno de juego. De foul y fault por contaminación.

$\mathbf{N}$

net $\underline{\mathrm{f} 1}$

Tejido hecho con hilos, cuerdas o material sintético, que se usa para dividir el área de ocupación de cada uno de los dos equipos que se enfrentan en un juego. /2 Falta que comete un jugador al tocar la red en su intento por pasar la pelota al lado contrario. / $\underline{3}$ Falta que decreta el árbitro cuando al sacar la pelota ésta roza la red [malla, red]. De net.

$\mathbf{R}$

réferi $\underline{m}$ Encargado de velar por el cumplimiento de las reglas del juego [árbitro, juez central]. De referee.

repris $\underline{m} \quad$ Repetición de una jugada cuando el partido se está transmitiendo por la televisión [repetición]. De reprise.

riley $\underline{\mathrm{m}} \quad$ Jugador que releva o sustituye a otro durante un partido [relevo, sustituto]. De relay.

\section{$\mathbf{S}$}

servicio $\underline{m} \quad$ Saque que hace un jugador para poner la pelota en movimiento e iniciar o continuar con el juego [saque]. De service.

set $\underline{\mathrm{m}}$

Suceso $\underline{m}$

V

volibol $\underline{m}$ Deporte colectivo jugado en un campo rectangular dividido por una red, entre dos equipos de seis jugadores, el cual consiste en golpear la pelota con la mano para pasarla por encima de la red, sin permitir que ésta toque el suelo [balonvolea]. De volleyball.

\section{Conclusiones y recomendaciones}

Realizada la investigación, hemos podido comprobar que:

1. La aportación léxica del inglés es muy importante en el campo del deporte, habiendo deparado un total de ciento cuatro (29) entradas y 124 acepciones.

2. La metodología empleada ha permitido un estudio científico del corpus.

3. Igualmente, se ha hecho evidente la estrecha relación lengua-cultura en el micro campo del deporte. 
La lengua como diasistema es permeable al cambio lingüístico, produciéndose las modificaciones de acuerdo con las necesidades comunicativas; el componente léxico, por su estrecha relación con el cambio de los objetos y referentes, se presenta como muy permeable al cambio lingüístico. Este hecho fundamenta una revisión del concepto purista de lengua.

Al finalizar la presente investigación nos hemos percatado de la necesidad de elaborar un diccionario costarricense, en el que, de ser posible, aparezca un registro léxico con diferentes micro campos. Comprendemos que realizar una labor de tal envergadura es sumamente costoso económicamente, tomaría mucho tiempo, además de la dedicación de un número considerable de investigadores. Una de las posibles maneras de simplificar en parte este trabajo es aprovechar las investigaciones lexicográficas que se han realizado en tesis de licenciatura y maestría, e incorporarlas al futuro diccionario costarricense.

\section{Bibliografía}

Alfaro, Ricardo. 1950. Diccionario de Anglicismos. Madrid: Editorial Gredos.

Carreter, Lázaro. 1969. Diccionario de Términos Filológicos. Madrid: Editorial Gredos.

Casares, Julio. 1942. Diccionario de la Lengua Española. Madrid: Editorial Gustavo Gil, S.A.

Corominas, J. 1980-1983. Diccionario Crítico Etimológico Castellano e Hispano. Madrid: Editorial Gredos S. A.

Cuervo, Rufino. 1983. Diccionario de Autoridades. Bogotá: Instituto Caro y Cuervo.

Cuyás, Arturo. 1980. Appleton's New English Spanish and Spanish English Dictionary. London: D. Appleton Century Co.

El Lenguaje (Diccionario de Lingüística). 1987. Bilbao: Ediciones Mensajero.

Enciclopedia Salvat de los Deportes. Volúmenes 1 y 5. 1987. Barcelona: Salvat Editores, S. A.

Ferrares, Juan Francisco. 1976. El Voleibol. Biblioteca Deportiva de Vecchi.

Gagini, Carlos. 1919. Diccionario de costarriqueñismos. San José, Costa Rica: Imprenta Nacional.

Haensch G., Wolf, L., S. Ettiner, y R. Werner. 1982. La Lexicografía. Madrid: Editorial Gredos.

1986. "La situación actual de la lexicografía del español de América". Revista de Filología Romántica, IV. Madrid: Editorial de la Universidad Complutense. 
1985. "La selección del material léxico para diccionarios descriptivos". En Philological Hispaniensia. In Honoren M1. Alvar. II Ling. Madrid: Edit. Gredos.

Lapesa, Rafael. 1942/1980. Historia de la Lengua Española. Madrid: Escelice.

Mora Cárdenas, Zuray. Cristian Rodríguez, los anglicismos y el español de Costa Rica. Tesis de licenciatura. Universidad de Costa Rica.

Pulido, A. 1985. Estadística y técnica de la investigación. Madrid: Ediciones Pirámide S. A.

Real Academia Española. 1986. Diccionario de la Lengua Española. España: Calpe, S. A. Vigésima Edición.

1973. Esbozo de una Nueva Gramática de la Lengua Castellana. Madrid: España Calpe.

Roget Mark, Peter. 1947. Thesaurus of Words and Phrases. New York: Crosset \& Dunlop.

Salas Madrigal, Agustín. 1951. Historia del Deporte en Costa Rica. Costa Rica: Imprenta Falcó.

Sánchez Corrales, Víctor. 1986. "Estudios en Costa Rica sobre lengua castellana: de Gagini a Agüero". Revista de Filología y Lingüística de la Universidad de Costa Rica. Vol. XII. (1).

1988. "Lexicografía del español en Costa Rica, visión crítica”. Revista de Filología y Lingüística de la Universidad de Costa Rica Vol. XIV (2).

Seco, Manuel. 1987. Estudios del lexicografía española. Madrid: Paraninfo S. A.

Spillner, Bernd. 1974. Lingüística y Literatura. Madrid: Editorial Gredos.

"V Copa Centroamericana Mayor de Voleibol”. 1989. Revista. San José, Costa Rica: Editorial Jiménez S. A.

Vox Diccionario Inglés-Español-Español-Inglés. 1970. Segunda Edición. Barcelona: Talleres Bibliográficos S.A.

Webster's New Twentieth Century Dictionary of the English Language Unabridged. 1971. Cleveland, Ohio: The World Publishing Company, Second Edition.

Wotjak, Gerd. 1977. Investigaciones sobre la estructura del significado. Madrid: Editorial Gredos. 
Zeloro Elías, Miguel de Toro y Emiliano Gómez. 1985. Diccionario del significado de la lengua Castellana. París: Garnier Hermanos, Editores.

Zúñiga Tristán, Virginia. 1976. El anglicismo en el habla costarricense. San Pedro de Montes de Oca: Editorial Universidad de Costa Rica. 